\title{
COMPARISON OF DOSE RATE WHEN WORKING WITH A C-ARM X-RAY SYSTEM
}

\author{
Natasha Ivanova ${ }^{1}$, Javor Ivanov ${ }^{2}$, Miroslav Petrov ${ }^{3}$, Marin Marinov ${ }^{4}$ \\ ${ }^{1}$ Department of Physics and Biophysics, Faculty of Pharmacy, Medical University of Varna \\ ${ }^{2} S \& T$ Bulgaria EOOD, Sofia \\ ${ }^{3}$ Clinic of Radiotherapy, St. Marina University Hospital, Medical University of Varna \\ ${ }^{4}$ Clinic of Orthopedics and Traumatology, St. Marina University Hospital, \\ Medical University of Varna
}

\begin{abstract}
INTRODUCTION: During working with sources of ionizing radiation, it is of special importance to take into account the dose load of the personnel working with such equipment.

AIM: The aim of this article is to discuss the dose load of people working with mobile X-ray systems with a C-arm, who perform orthopedic procedures in the Clinic of Orthopedics and Traumatology of the St. Marina University Hospital, Varna. This research tries to convincingly show orthopedic surgeons and traumatologists that using a fluoroscopy mode (in all its variations) of the $\mathrm{C}$-arm $\mathrm{X}$-ray system when the tube is positioned under the patient table is best for their work when it comes to the working conditions, the image quality, the information value and the dose exposure of the staff.

MATERIALS AND METHODS: In the article a comparison of the dose load (effective dose) was made based on dosimetric measurements with respect to the personnel in different modes of operation of the X-ray equipment-roentgenoscopy (single shot) and fluoroscopy. The doses received by the surgeon-operator and his assistant were measured at three points of the body-head, gonads and feet, in radiography mode (single shot) and in three sub-modes of the fluoroscopy mode-continuous fluoroscopy, $1 / 2$ dose fluoroscopy, and pulse fluoroscopy.

For a more accurate comparison of the dose load in the respective operating modes, the time for reaching the dose limit for the personnel, determined by the Bulgarian legislation, was calculated-namely $20 \mathrm{mSv}$ per year.

RESULTS: The results of all measurements and calculations showed that the dose load in the radiography mode (single shot) was several times larger than the dose that was received in the three variations of the fluoroscopy mode at the same operating time.
\end{abstract}

\footnotetext{
Address for correspondence:

Natasha Ivanova

Faculty of Pharmacy

Medical University of Varna

84 Tzar Osvoboditel Blvd

9002 Varna

e-mail:natasha_i@abv.bg
}

Received: November 8, 2020

Accepted: December 15, 2020
CONCLUSION: The results suggest that when using the fluoroscopy mode, even in the sub-mode with the highest dose load, it gives a lower dose than using the mode roentgenography, at the same time of operation of the X-ray tube.

Keywords: C-arm X-ray equipment, dose load, staff, Orthopedics and Traumatology Clinic 


\section{INTRODUCTION}

Our study examines the dose load received by the staff working with the C-arm X-ray system at the Clinic of Orthopedics and Traumatology at the St. Marina University Hospital, Varna. The X-ray equipment in the clinic has two main modes of operation of the X-ray system used for orthopedic procedures, namely:

$\diamond$ radiography (single shot)_during this mode an image (roentgen photos) of the region of interest is made. This mode has variations for different parts of the body, with the corresponding factory-set parameters for the X-ray tube: for examination of the shoulder, wrist, lower leg, foot, etc.

$\diamond$ fluoroscopy-during this mode the object is observed in real time.

The fluoroscopy modes have two variations: LDF (low-dose fluoroscopy) and HDF (high-resolution fluoroscopy). Each of them has three submodes: continuous fluoroscopy; $1 / 2$ dose fluoroscopy and pulse fluoroscopy $(1,2)$.

Examining the two modes, we aim to make a comparison between the dose load of the personnel received from these two regimes for the same working time.

There are two main operating modes of the Xray system used for orthopedic procedures, namely:

$\diamond$ radiography (single shot)-here a single image is taken of the region of interest; this mode has variations for different parts of the body, with respective factory-set parameters for the X-ray tube: for examination of shoulder, wrist, lower leg, foot, etc.

$\diamond$ fluoroscopy-here the object is observed in real time: the fluoroscopy modes have two variations: LDF-low-dose fluoroscopy и $\mathrm{HDF}-$ high-definition fluoroscopy. Each of them has three submodes: continuous, $1 / 2$ dose, and pulse $(1,2)$.

\section{AIM}

The aim of this article is to discuss the dose load of people working with mobile X-ray systems with a C-arm, who perform orthopedic procedures in the Clinic of Orthopedics and Traumatology of the St. Marina University Hospital, Varna. This research tries to convincingly show orthopedic surgeons and traumatologists that using a fluoroscopy mode (in all its variations) of the $\mathrm{C}$-arm X-ray system when the tube is positioned under the patient table is best for their work when it comes to the working conditions, the image quality, the information value and the dose exposure of the staff.

\section{MATERIALS AND METHODS}

\section{Dosimetric Measurement}

We measured the values of the dose received by the operator and his assistant at three points on their bodies-head, gonads and feet, in both modes of operation of the C-arm.

Based on the obtained dose values, the dose rates for each of the operating modes and all measured points of the body of the operator and his assistant were calculated. Dose rate calculation was necessary because the exposure time was different for each mode.

We also calculated the time to reach the maximum annual dose allowed for staff, which is $20 \mathrm{mSv}$, as stipulated in the Bulgarian legislation. The values from this calculation showed the results of the measurements more clearly.

The practical measurements were made in the Clinic of Orthopedics and Traumatology of St. Marina University Hospital, Varna, Bulgaria.

Participants in the dosimetric measurement:

$\diamond$ first author of the article-N. Ivanova, Department Physics and biophysics, Medical University, Varna,

$\diamond$ service engineer maintaining the X-ray equipment-the measurements were carried out under the direct and continuous control of the service engineer of the $\mathrm{X}$-ray system,

$\diamond$ medical physicist of the hospital who works with the measuring equipment,

$\diamond$ surgeon-a specialist from the clinic of orthopedics and traumatology with instructions about the used medical procedures.

For our measurements we used an X-ray system type C-arm Phillips BV Vectra located in the Clinic of Orthopedics and Traumatology (Fig. 1).

For the measurements, a digital dose meter of the type FH 40 G-L (Fig. 2) was used. The Thermo Scientific $^{\text {Ta }}$ FH 40 G-L is a wide-range digital Geiger counter suitable for nearly all measurement tasks 
arising in radiation protection (3). For the measurements, we used a phantom. For the phantom, we used a plastic bottle with a volume of 11 liters filled with water (Fig. 3). The phantom was placed on the patient's table, directly under the X-ray beam (2).
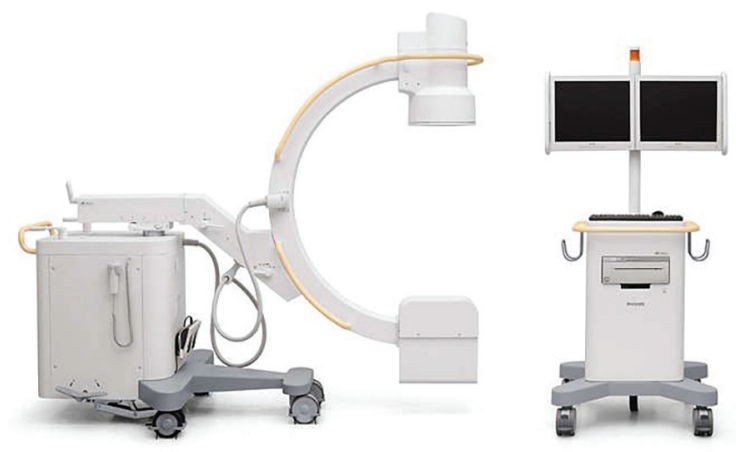

Fig. 1. X-ray system BV Vectra-mobile C-arm (1).

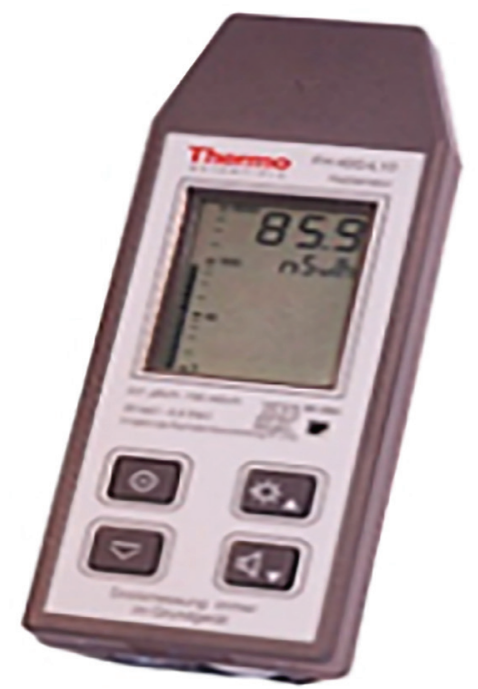

Fig. 2. FH 40 G-L multi-purpose digital survey meter (3)

During our measurements, we irradiated the phantom using the two main modes of the C-arm radiography (single shot) and LDF. We used the three modes of fluoroscopy - continuous fluoroscopy, $1 / 2$ dose fluoroscopy, and pulse fluoroscopy.

The operating parameters under which the study was performed were:

$\diamond$ radiography (single shot); X-ray tube parameters: $\mathrm{U}=58 \mathrm{kV}$; I.t $=10.35 \mathrm{mAs}$; duration of the single shot, $\mathrm{t}=467 \mathrm{msec}$.

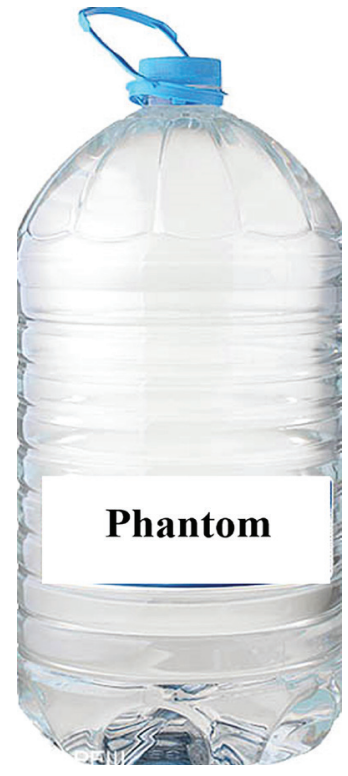

Fig. 3. Phantom

$\diamond$ fluoroscopy: continuous fluoroscopy; X-ray tube parameters: $\mathrm{U}=58 \mathrm{kV}$; I.t $=3.45 \mathrm{mAs}$; exposure time $t=3 \mathrm{sec}$., $1 / 2$ dose fluoroscopy; $X$ ray tube parameters: $\mathrm{U}=58 \mathrm{kV}$; I.t $=3.45 \mathrm{mAs}$; exposure time $\mathrm{t}=6 \mathrm{sec}$., pulse fluoroscopy; X-ray tube parameters: $\mathrm{U}=58 \mathrm{kV}$; I.t $=3.45 \mathrm{mAs}$; exposure time $\mathrm{t}=6 \mathrm{sec}$.

The position of the $\mathrm{C}$-arm was perpendicular to the patient table, with the X-ray tube placed below the patient table and the image intensifier above it. The distance between the X-ray tube and the patient was $70 \mathrm{~cm}$. The distance between the $\mathrm{C}$-arm axis (the axis connecting the X-ray tube and the image intensifier) and the surgeon was $60 \mathrm{~cm}$, and to his assistant-100 $\mathrm{cm}$. The surgeon was sitting (as close as possible to the patient), and the assistant stayed upright. Measurements were made for three points of the bodies of the personnel working with the equipment: head, gonads, and feet (Fig. 4). When using the radiography mode, the mobile station can be set to directly produce pictures of a specific part of the body: arm, leg, chest, etc. Each of these submodes has specific preset $\mathrm{kV}$ and $\mathrm{mAs}$, which cannot be changed during the radiography examination. These preset parameters are in accordance with the average parameters of the examined object (size, shape, density, etc.).

However, if the properties of the irradiated object differ significantly from these averages (for example, a hand of a small child or a fairly obese pa- 


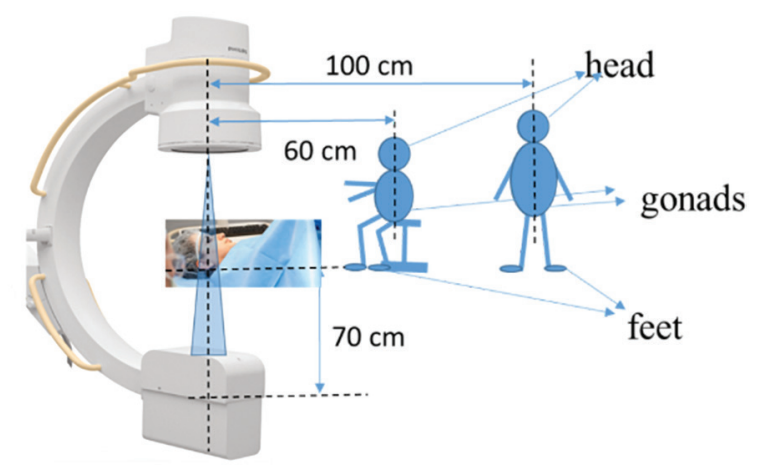

Fig. 4. Positions and distances during the study.

tient), the picture is not of high quality and the information value of the image is reduced.

When using the fluoroscopy mode, the X-ray tube starts an automatic mode in which, on the basis of the data obtained from the irradiation of the object (shape, size, density, etc.), it sets optimal values of $\mathrm{mAs}$ and $\mathrm{kV}$. In this way, a high quality and informative image of the irradiated object is obtained. If the system is used in X-ray mode after using fluoroscopy mode, the data collected from fluorography will be used (this is a factory setting). So, in radiography mode, the optimal parameters are used in order to get the best image of the region of interest of the respective patient (1).

No members of the personnel and the research team were present during our measurements in the procedure room for reasons of radiation protection. The door to the room was left open, and the team stood behind the wall of the room to ensure maximum protection from scattered radiation. Only the $\mathrm{C}$-arm stand remained in the procedure room. The station with the monitors was placed in the corridor outside the procedure room. The measuring device was placed on a stand that could be moved horizontally and vertically. In this way, the required measuring point could be selected.

Due to the fact that the measurement was carried out with a phantom that was of smaller size than the normal human body and that there were no personnel in the treatment room, it was expected that the scattered radiation was lower. This difference was not great and therefore, we could apply these results to real situations as well (2).

\section{RESULTS}

At different positions of the X-ray tube, 15 to 20 repetitions of measurements were made for the same parameters of the X-ray tube.

For better clarity, we calculated the time for continuous operation of the X-ray system needed to reach the limit of $20 \mu \mathrm{Sv}$ for one calendar year (stipulated by the Bulgarian legislation) on the basis of the average values of the measurements. The data for the surgeon-operator are shown in Table 1 and Diagram 1 , and for his assistant in Table 2 and Diagram 2. It is clear from the diagrams that the time to reach the dose limit for both the surgeon and his assistant differs greatly in the radiographic and the fluoroscopic modes.

The time for reaching the annual dose limit for the surgeon in the measurement point "gonads" (Diagram 1-the most unfavorable radiation-loaded point) for radiography mode was only 3.6 days of continuous work. At the same time, even for the heaviest mode of fluoroscopy, namely continuous fluoroscopy, this time was 23.2 days (hence, the time is 7 times greater). The other two fluoroscopic submodes showed even longer times.

For the point of the lowest dose, namely "feet," this time for the surgeon (Diagram 1) was 4.7 days for radiography mode. For continuous fluorography it was 34.5 days (an increase of almost 7 times); for the two fluoroscopy submodes it was about 15 times larger (Diagram 1).

The comparison in the Diagram 2 for the times to reach the annual dose limits for the assistant shows similar results. For point "gonads" this time was 7.7 days, for continuous fluoroscopy the time was 62.5 days. and for $1 / 2$ dose fluoroscopy and pulse fluoroscopy it was 163 days and 193, respectively (almost 25 times longer) (Diagram 2).

The calculated time for the point "feet," where the dose load is lowest for the radiography mode, was 9.8 days and for continuous fluoroscopy it was 116 days (12 times longer). When using $1 / 2$ dose fluoroscopy, the time was increased 17 times (163 days). For the pulse fluoroscopy mode this time increased 30 times (289 days) (Diagram 2). 
Comparison of Dose Rate When Working with a C-Arm X-Ray System

Table 1. Time until reaching the annual limit dose value of $20 \mu S v$ for different points of the surgeon's body

\begin{tabular}{|c|c|c|c|c|}
\hline \multicolumn{5}{|c|}{ Surgeon } \\
\hline Measurement point & Time & Head & Gonads & Feet \\
\hline \multirow{3}{*}{ Single shot } & seconds & 359066 & 311327 & 406504 \\
\hline & hours & 100 & 86 & 113 \\
\hline & days & 4.1 & 3.6 & 4.7 \\
\hline \multirow{3}{*}{ Continuous fluoroscopy } & seconds & 2857143 & 2000000 & 2985075 \\
\hline & hours & 794 & 556 & 829 \\
\hline & days & 33 & 23.2 & 34.5 \\
\hline \multirow{3}{*}{$1 / 2$ dose fluoroscopy } & seconds & 5714286 & 4166667 & 6060606 \\
\hline & hours & 1587 & 1157 & 1684 \\
\hline & days & 66.1 & 48.2 & 70.2 \\
\hline \multirow{3}{*}{ Pulse fluoroscopy } & seconds & 6060606 & 4000000 & 6250000 \\
\hline & hours & 1684 & 1111 & 1736 \\
\hline & days & 70.2 & 46.3 & 72.3 \\
\hline
\end{tabular}

Table 2. Time until reaching the annual limit dose value of $20 \mu S v$ for different points of the assistant s body

\begin{tabular}{|c|c|c|c|c|}
\hline \multicolumn{5}{|c|}{ Assistant } \\
\hline Measurement point & Time & Head & Gonads & Feet \\
\hline \multirow{3}{*}{ Single shot } & seconds & 778210 & 666667 & 847458 \\
\hline & hours & 216 & 185 & 235 \\
\hline & days & 9 & 7.7 & 9.8 \\
\hline \multirow{3}{*}{ Continuous fluoroscopy } & seconds & 8695652 & 5405405 & 10000000 \\
\hline & hours & 2415 & 1501 & 2778 \\
\hline & days & 100.6 & 62.5 & 115.75 \\
\hline \multirow{3}{*}{$1 / 2$ dose fluoroscopy } & seconds & 16666667 & 11764706 & 25000000 \\
\hline & hours & 4639 & 3268 & 6944 \\
\hline & days & 193 & 163 & 289 \\
\hline \multirow{3}{*}{ Pulse fluoroscopy } & seconds & 20000000 & 16666667 & 25000000 \\
\hline & hours & 5556 & 4630 & 6944 \\
\hline & days & 232 & 193 & 289 \\
\hline
\end{tabular}

\section{DISCUSSION}

The maximum possible reduction of the received dose for the personnel working with ionizing radiation is a very important problem in modern world. While the patient receives a dose only during the procedure, the staff working with the equip- ment receives a dose (from the scattered X-ray radiation) every day during work. This is the reason why we focused our efforts on studying the dose load of personnel working with a C-arm X-ray system. This type of X-ray equipment can be used for life-saving procedures in various fields of medicine, such as or- 


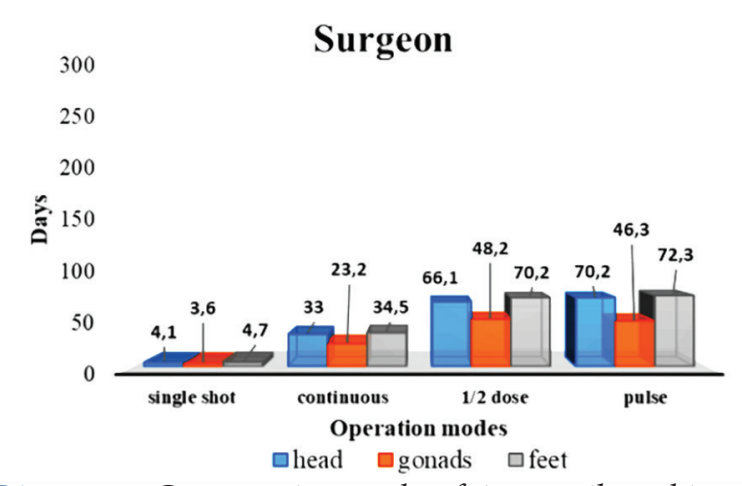

Diagram 1. Comparative graphs of time until reaching the annual limit dose value of $20 \mu S v$ for different points of the surgeon's body

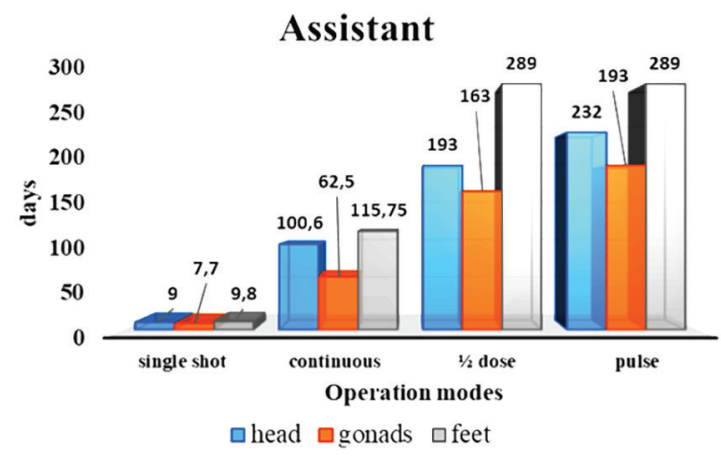

Diagram 2. Comparative graphs of time until reaching the annual limit dose value of $20 \mu \mathrm{Sv}$ for different points of the assistant's body

thopedics and traumatology, surgery, urology, cardiology, and others. Flexibility in choosing the direction of the imaging, as well as preserving the geometric dimensions in different directions, is achieved by using the constructive solution of the $\mathrm{C}$-arm X-ray equipment (1).

The radiation protection from scattered radiation from the irradiated body, the patient table, the housing of the equipment and all other objects in the room is very important for maintaining the health and life of people working in an environment with ionizing radiation.

The research in this direction is urgent and necessary.

The purpose of the work of C. Guo and his coauthors is to develop a database of $3 \mathrm{D}$ scattered radiation dose-rate distributions to estimate the staff dose by location around a C-arm fluoroscopic system in an interventional procedure room. The results show that the shape of the phantom will affect the dose-rate distribution at distances less than 100 $\mathrm{cm}$, with a higher intensity for the super-ellipse. This database allows estimation of the scattered radiation distribution in the procedure room and, when displayed to the staff during a procedure, may facilitate a reduction of occupational dose (4).

In the paper „Radiation Exposure to Operating Room Personnel and Patients During Endovascular Procedure," Abhisekh Mohapatra and his colleagues measured and discussed the dose received by patients and staff during endovascular procedures using the fluoroscopic mode of the X-ray system (5). W.H. Bush and his colleagues measure and discusse the dose received by the staff during percutaneous removal of renal calculus by using the fluoroscopic mode of the C-arm system. The measurement is made only at one point-at the collar level above the lead apron. The article examines the effectiveness of individual radiation protection of a personnel (6).

In the articles „Real-Time, Ray Casting-Based Scatter Dose Estimation for C-Arm X-Ray System“ (7) and „Radiation Doses in Interventional Radiology Procedures: The RAD-IR Study: Part II: Skin Dose" (8) the received dose in the patient's skin during various procedures using $\mathrm{C}$-arm in fluoroscopic mode is discussed. In the first article a phantom was used and in the second article real data from procedures with patients were collected. Each of these articles comments on the dose load in different $\mathrm{C}$-arm procedures and ultimately aims at better radiation protection for both staff and patients.

Our study described here has the same ultimate goal. One of the main goals of our study is to compare the dose load (based on the effective dose obtained) for the two modes of operation of the C-arm-radiography and fluoroscopy. Due to the fact that there are three submodes in fluoroscopy mode, we compare the dose load for all three submodes. Our study focuses on the use of $\mathrm{C}$-arm for orthopedic procedures. We also examine the dose received by the patient and the dose received by the staff.

Specifically, in this article we give a comparison of the dose load of the staff in the two main modes of operation of the C-arm, at three points of the body of the surgeon and his assistant-head, gonads, and feet. The dosimetric control performed by the con- 
trol authorities is done in these three points. For this reason, we also used them in our study.

\section{CONCLUSION}

We made the comparison between the different modes of operation of the X-ray tube in three different ways, but here in this article we consider only one of them: by calculating the time at the measured points, which would be necessary to reach the dose limit of $20 \mu \mathrm{Sv}$ for the personnel according to the Bulgarian legislation.

If one compares the times to reach the annual limit value for the dose exposure in Tables 1 and 2 (or in Diagrams 1 and 2), it is found that the fluoroscopy mode is at least seven times more favorable than the radiography mode. This can be seen from Diagrams 1 and 2, which show a large time difference.

While in the radiography mode for the surgeon (at all measured points), the time to reach the dose limit is about half a week, in the $1 / 2$ fluoroscopy mode, this time is about a month and a half. The difference is significant. In the same comparison for the assistant in radiography mode (at all measured points) the time is about a week and a half, in the 1/2 fluoroscopy mode this time is almost one year. Here the difference is significant.

The main conclusion of our study is that fluoroscopy mode is more favorable for work with the same exposure time.

The images obtained from the above-described modes of operation with the relevant parameters were informative enough for the purposes of the respective medical examination, according to the opinion of the orthopedist in our research team. In this case, it can be concluded that the single shot radiography mode can be replaced by fluoroscopy (in all three variations) for some period of time, while maintaining the required information value and image quality, and sometimes even higher image quality.

\section{REFERENCES}

1. BV Vectra, Instructions for use. Document version 1.4. Philips India Limited, Plot No. B-79, MIDC, Phase-II, Chakan Taluka - khed, Village - Savardari, District: Pune, Maharashtra 410501, India, Issue Number 459800632434.

2. Ivanova N. C-arm X-ray system. Dose load of personnel and patients. Medical University of Varna; 2020. (in Bulgarian).

3. Thermo Fisher Scientific. Available at: https://www.thermofisher.com/order/catalog/ product/4254002\#/4254002

4. Guo C, Xiong Z, Vijayan S, Rudin S, Bednarek DR. Developing a database of 3-D scattered radiation distributions for a c-arm fluoroscope as a function of exposure parameters and phantom. Proc SPIE Int Soc Opt Eng. 2018;10573:1057360. doi: $10.1117 / 12.2293788$

5. Mohapatra A, Greenberg RK, Mastracci TM, Eagleton MJ, Thornsberry B. Radiation exposure to operating room personnel and patients during endovascular procedures. J Vasc Surg. 2013;58(3):702 9. doi: 10.1016/j.jvs.2013.02.032.

6. Bush WH, Jones D, Brannen GE. Radiation dose to personnel during percutaneous renal calculus removal. AJR Am J Roentgenol. 1985;145(6):1261-4. doi: 10.2214/ajr.145.6.1261.

7. Alnewaini Z, Langer E, Schaber P, David M, Kretz $\mathrm{D}$, Steil V, et al. Real-time, ray casting-based scatter dose estimation for c-arm X-ray system. J Appl Clin Med Phys. 2017;18(2):144-153. doi: 10.1002/ acm2.12036.

8. Miller DL, Balter S, Cole PE, Lu HT, Berenstein A, Albert R, et al. Radiation doses in interventional radiology procedures: the RAD-IR study: part II: skin dose. J Vasc Interv Radiol. 2003;14(8):977-90. doi: 10.1097/01.rvi.0000084601.43811.cb. 